Service social

\title{
Placement en famille d'accueil. Étude sur les variables associées aux contacts entre l'enfant placé et ses parents
}

\section{Marie-Andrée Poirier, Marie Simard et Jacques Vachon}

Volume 47, numéro 3-4, 1998

Enfance : enjeux et réalités 2000

URI : https://id.erudit.org/iderudit/706797ar

DOI : https://doi.org/10.7202/706797ar

Aller au sommaire du numéro

Éditeur(s)

École de service social de l'Université Laval

ISSN

1708-1734 (numérique)

Découvrir la revue

Citer cet article

Poirier, M.-A., Simard, M. \& Vachon, J. (1998). Placement en famille d'accueil. Étude sur les variables associées aux contacts entre l'enfant placé et ses parents. Service social, 47(3-4), 115-136. https://doi.org/10.7202/706797ar
Résumé de l'article

Cette étude porte sur le maintien des liens entre l'enfant placé et ses parents. Elle a pour objectif de connaître la fréquence et la durée des visites chez ses parents de l'enfant placé et d'identifier les variables pouvant y être associées. Les données ont été recueillies à l'aide d'un questionnaire administré par entrevue auprès de 58 parents ayant un enfant placé en famille d'accueil régulière depuis au moins six mois et ayant des contacts non supervisés avec ce dernier. Les parents furent sélectionnés parmi la clientèle de quatre centres jeunesse au Québec. Les résultats indiquent que $43 \%$ des parents reçoivent la visite de leur enfant plus de deux fois par mois et que les enfants passent en moyenne quatre jours par mois chez leurs parents. Les principales variables associées à la fréquence et à la durée des visites de l'enfant chezses parents sont le temps de transport, les raisons de placement, la séparation des membres de la fratrie dans des milieux d'accueil différents et la prévision ou non du retour de l'enfant dans son milieu familial. 


\title{
Placement en famille d'accueil Étude sur les variables associées aux contacts entre l'enfant placé et ses parents
}

\author{
Marie-Andrée POIRIER \\ Marie SIMARD \\ Jacques VACHON
}

Cette étude porte sur le maintien des liens entre l'enfant placé et ses parents. Elle a pour objectif de connaître la fréquence et la durée des visites chez ses parents de l'enfant placé et d'identifier les variables pouvant y être associées. Les données ont été recueillies à l'aide d'un questionnaire administré par entrevue auprès de 58 parents ayant un enfant placé en famille d'accueil régulière depuis au moins six mois et ayant des contacts non supervisés avec ce dernier. Les parents furent sélectionnés parmi la clientèle de quatre centres jeunesse au Québec. Les résultats indiquent que $43 \%$ des parents reçoivent la visite de leur enfant plus de deux fois par mois et que les enfants passent en moyenne quatre jours par mois chez leurs parents. Les principales variables associées à la fréquence et à la durée des visites de l'enfant chez ses parents sont le temps de transport, les raisons de placement, la séparation des membres de la fratrie dans des milieux d'accueil différents et la prévision ou non du retour de l'enfant dans son milieu familial.

This article presents the results of a study on visits between parents and children placed in family foster care. The aims of this study are:1) describe the frequency and length of children's visits with their parents and 2) identify the variables associated with the frequency and length of visits. Fifty-eight parents who have a child placed in family foster care for at least six months and who have non-supervised contacts with the child were interviewed. The data were collected with a face-to-face questionnaire. The results show that $43 \%$ of the parents had contact with their child more than two times a month and the children spent an average of 4 days a month with their parents. The variables associated with the frequency and length of visits are the time of transport between the foster family's home and the parent's home, the reason of placement, the separation of siblings in more than one foster families and the presence of a reunification plan. 


\section{INTRODUCTION}

Depuis de nombreuses années, dans le domaine des services sociaux, on confie des enfants à une ressource d'accueil afin d'assurer leur bien-être et leur protection. Ces enfants sont généralement retirés de leur milieu familial en raison de négligence, d'abus ou de problèmes de comportement. Au Québec, au 31 mars 1998, on comptait environ 12000 enfants placés, dont 8270 en famille d'accueil et 3730 en ressources institutionnelles (Gouvernement du Québec, 1998). Selon Lessard (2000), 35,4 \% des nouvelles prises en charge en protection de la jeunesse en 1998-1999 impliquaient une mesure de placement en milieu substitut.

Dans les études cliniques et empiriques menées dans le domaine du placement le maintien des liens entre l'enfant placé et ses parents apparaît comme une variable importante. En effet, de nombreuses recherches identifient le maintien des liens comme l'un des principaux facteurs de réussite de la réinsertion familiale (Davies, Landsverk, Newton et Ganger, 1996; Simard, Vachon et Tard, 1991). On souligne également que les enfants qui maintiennent des contacts avec leurs parents s'adaptent davantage à leur situation de placement (Bullock, Hosie, Little et Millham, 1990; Cantos, Gries et Slis, 1997), fonctionnent mieux lors du retour dans leur famille (Fein, Maluccio, Hamilton et Ward, 1983) et risquent moins de vivre un nouveau placement (Block et Libowitz, 1983).

En raison de l'importance du maintien des liens entre l'enfant placé et ses parents, de nombreux travaux ont été menés au cours des dernières années afin de comprendre les variables qui y sont associées. Les variables identifiées dans ces études sont principalement reliées aux caractéristiques des familles, des parents, des enfants, des situations de placement et de l'intervention'.

Les études montrent que les familles qui visitent moins leur enfant proviennent d'un milieu socio-économique plus faible (Jenkins et

1 Le lecteur intéressé peut consulter une recension des écrits sur le sujet publiée par l'auteure: M.-A. Poirier, « Le maintien des liens entre l'enfant placé et ses parents. Analyse critique de travaux de recherche ", Revue canadienne de service social, 1998, vol. 15, $\mathrm{n}^{\circ}$ 1, p. 9-24. 
Norman, 1972 et Vachon, 1978) ${ }^{2}$, éprouvent des difficultés financières pour assurer le transport (Jenkins et Norman, 1975; Fanshel, 1982; Kufeldt et collab., 1989, 1996), présentent des problèmes de santé physique ou mentale (Jenkins et Norman, 1975; Fanshel, 1982; Kufeldt et collab., 1996) et assument d'autres responsabilités parentales (Fanshel, 1982).

On note des résultats contradictoires quant à la relation observée entre l'âge de l'enfant placé et la fréquence des visites. Dans les études de Fanshel et Shinn (1978), Jenkins et Norman (1972), Richardson, Galaway, Hudson, Nutter et Hill (1995) et Vachon (1978), les enfants plus âgés sont plus souvent visités. Par contre, d'autres auteurs (O'Higgins, 1996; Oyserman et Bebenshity, 1992) arrivent à la conclusion inverse: les enfants plus jeunes sont davantage visités par leurs parents. De plus, l'étude québécoise de Vachon (1978) montre que les filles sont plus visitées que les garçons, alors que les chercheurs Richardson et collab. (1995) n'observent aucune différence significative entre les garçons et les filles sur ce plan.

Un constat se dégage des études recensées : les enfants les plus visités sont ceux placés depuis moins longtemps (Bilson et Barker, 1995; Cantos, Gries et Slis, 1997; Fanshel et Shinn, 1978; Fanshel, 1982; Mech, 1985; Palmer, 1992). Palmer (1992) mentionne également que les enfants qui ont vécu plus d'un placement sont moins visités par leurs parents. Pour leur part, Richardson et collab. (1995) soulignent que les enfants qui vivaient avec leurs parents avant le placement sont plus visités

2 L'étude de Vachon (1978) constitue une vérification de l'étude de Jenkins et Norman (1972). C'est la seule étude québécoise qui a examiné les contacts entre les parents et leurs enfants placés. L'étude porte sur 104 mères dont les enfants sont placés en famille d'accueil dans la région de Montréal.

Dans ces deux études le niveau socio-économique des familles a été calculé à l'aide d'un index qui tient compte de la source de revenu, du niveau de scolarité, du revenu moyen et de la délinquance juvénile dans la zone de résidence (pour l'étude de Jenkins et Norman, 1972) ainsi que des conditions de logement. Cet index a permis de diviser les familles en trois groupes selon leur statut socio-économique (haut, moyen, bas). Selon les auteurs de ces études, cette façon de procéder permet de discriminer les familles dans un échantillon relativement homogène sur le plan du revenu, mais rend difficile la comparaison avec d'autres travaux. 
que ceux qui proviennent d'un autre milieu d'accueil. Les résultats de l'étude de Fanshel (1982) indiquent une fréquence plus élevée de contacts avec les parents lorsqu'on prévoit le retour de l'enfant dans son milieu familial. On observe dans plusieurs études que les enfants placés pour des problèmes de comportement ou pour des problèmes émotifs sont les plus visités et que les enfants placés pour abus/négligence ou pour abandon sont les moins visités (Bilson et Barker, 1995; Fanshel et Shinn, 1978; Jenkins et Norman, 1972, 1975; Vachon, 1978). La grande distance géographique entre le milieu d'accueil et le milieu familial (Cliffe et Berridge, 1991; Kufeldt et collab., 1989, 1996) est également considérée comme un obstacle important dans la mise en place des visites.

Finalement, de nombreux travaux montrent que les parents qui ont des contacts fréquents avec l'intervenant visitent plus régulièrement leur enfant placé (Fanshel et Shinn, 1978; Gillespie, Byrne et Workman, 1995; Newcomb, 1986).

De façon générale, les études recensées permettent d'avoir un portrait des différentes variables associées à une plus ou moins grande fréquence de contacts entre l'enfant placé et ses parents. Toutefois, certaines caractéristiques ou limites de ces études diminuent la portée de leurs résultats. Premièrement, la diversité des échantillons présentés dans chacune des études (âge des enfants, contexte de placement, programmes particuliers) rend difficile la comparaison des résultats d'une recherche à l'autre. Deuxièmement, on observe une différence importante entre les études quant à la source d'information. Certains chercheurs ont recours aux dossiers (Mech, 1985; Oyserman et Bebenshity, 1992; Palmer, 1992; Richardson et collab., 1995). D'autres utilisent comme informateur soit l'intervenant (Bilson et Barker, 1995; Fanshel et Shinn, 1978; Fanshel, 1982; Hess, 1988; Ravier, 1995), soit le parent d'accueil (Berrick et collab., 1994; Butler, 1982; Cantos et collab., 1997). Peu de travaux s'appuient sur le point de vue des parents (Jenkins et Norman, 1972, 1975; Vachon, 1978). Troisièmement, malgré la convergence des études concernant certaines variables associées à la fréquence des contacts parents/ enfant (l'état de santé des parents, les problèmes de transport, la durée de placement et le type de milieu d'accueil), pour d'autres variables il subsiste certains résultats contradictoires qui ont trait à 
l'influence des variables telles que la présence d'autres responsabilités familiales et l'âge des enfants. Finalement, on peut noter que certaines variables sont peu ou pas prises en considération (la présence de plusieurs enfants placés, les réactions des parents au placement ainsi que leurs difficultés personnelles et parentales).

Afin de combler certaines de ces lacunes, nous avons réalisé une étude portant sur la perspective des parents concernant le maintien des liens lors du placement d'un enfant en famille d'accueil ${ }^{3}$. L'objectif de cet article est de présenter les résultats portant sur la fréquence et la durée des visites de l'enfant dans son milieu familial et les variables qui y sont associées.

\section{MÉTHOdOLOGIE}

Les deux variables dépendantes considérées dans cette étude sont la fréquence et la durée des visites de l'enfant chez ses parents ${ }^{4}$. Un grand nombre de variables indépendantes ont été retenues dans l'étude afin de prendre en considération la complexité des situations de placement; elles ont trait aux caractéristiques des parents, des familles, des enfants, de leur histoire de placement, du placement actuel et de l'intervention (voir tableau 1).

Nous avons interrogé 58 parents (46 mères et 12 pères) $)^{5}$ ayant au moins un enfant placé (de moins de 14 ans) en famille d'accueil régulière par un centre jeunesse - Estrie, Montérégie, Québec et Chaudière-Appalaches - et qui ont eu au moins un contact non supervisé avec leur enfant placé au cours des six mois précédant la collecte des données. Celle-ci a été réalisée à l'aide d'un questionnaire administré sous forme d'entrevue en face à face au

${ }^{3}$ Ces résultats sont tirés d'une étude sur l'implication parentale lors du placement d'un enfant en famille d'accueil menée par l'auteure. Voir M.-A. Poirier, L'implication parentale lors du placement d'un enfant en famille d'accueil. La perspective des parents. Thèse de doctorat non publiée, Québec, École de service social, Université Laval, 2000.

4 Dans les situations où les parents sont séparés, l'étude ne porte que sur le parent le plus souvent en contact avec l'enfant.

5 Nous devons préciser que les parent ont été recrutés sur une base volontaire. 
domicile des parents. La durée moyenne de l'entrevue était de soixante minutes.

\section{Tableau 1}

Variables indépendantes

\begin{tabular}{ll}
\hline \multicolumn{1}{c}{ Catégories } & Variables \\
\hline Caractéristiques des & Âge \\
parents & Scolarité \\
& $\begin{array}{l}\text { Présence d'un conjoint ou d'une conjointe } \\
\text { Statut d'emploi } \\
\text { Source de revenu } \\
\text { Présence de certains problèmes } \\
\text { personnels (santé physique ou mentale, } \\
\text { alcoolisme ou toxicomanie) }\end{array}$ \\
& Revenu annuel familial \\
Caractéristiques des & Nombre d'enfants \\
familles & Nombre d'enfants placés \\
& Nombre de milieux d'accueil avec lesquels \\
& le parent est en contact \\
\hline Caractéristiques des & Âge \\
enfants et de leur & Sexe \\
histoire de placement & Nombre de placements vécus depuis la \\
& naissance \\
\hline Caractéristiques de la & Principale raison de placement selon le \\
parent \\
placement
\end{tabular}


Les données de l'étude ont été traitées et analysées au moyen du progiciel SPSS (version 9.0). En premier lieu, des analyses descriptives (distribution de fréquences et statistiques descriptives) ont été effectuées pour chacune des variables de l'étude afin de dresser un portrait des caractéristiques des parents, des familles, des enfants et des situations de placement qui composent l'échantillon. En second lieu, des analyses ont été réalisées afin de vérifier l'existence d'une association entre les différentes variables indépendantes et la fréquence ainsi que la durée des visites de l'enfant chez ses parents. Le choix du test statistique (chi carré, test $t$, analyses de corrélation) a été fait en fonction de la nature des variables étudiées. Nous avons conclu à l'existence d'une association lorsque le degré de signification du test statistique est égal ou en deçà de 0,05 . Finalement, des analyses de régression multiple ont été effectuées afin de développer des modèles (un ensemble de variables indépendantes) qui permettent de prédire la fréquence et la durée des visites de l'enfant chez ses parents.

\section{Portrait de l'échantillon}

L'âge moyen des parents rencontrés est de 35,6 ans (écart-type = 5,2 ). Ces parents ont en moyenne 11 ans de scolarité (écart-type $=2,2$ ) et près de la moitié $(48,3 \%)$ n'ont pas fait leur $5^{\mathrm{e}}$ secondaire. Trente et un pour cent des parents rapportent avoir des problèmes de santé physique, près de $30 \%$ des problèmes de santé mentale ou des problèmes émotifs et environ $10 \%$ des problèmes de consommation d'alcool, de drogue ou de médicaments. Seulement $17,2 \%$ des parents occupent un emploi rémunéré, la majorité vivent de la sécurité du revenu $(79,3 \%)$ et $86,3 \%$ rapportent un revenu familial de 19999 \$ et moins. La majorité des parents sont chefs de famille monoparentale $(60,3 \%)$ et ont en moyenne 2,7 enfants (écart-type de 1,5$)$ dont l'âge moyen est de 9,4 ans (écart-type de 3,4 ans). Plus de la moitié des parents $(54,4 \%)$ ont deux enfants placés et plus. Soulignons que dans $35,5 \%$ des cas les enfants sont placés dans des ressources d'accueil différentes. 
Le nombre d'enfants inclus dans l'étude est de $56^{6}$, soit 39 garçons et 17 filles. L'âge moyen des enfants est de 9,2 ans (écarttype $=2,7$ ). La moitié des enfants vivent leur première expérience de placement. Toutefois, pour 33,9\% d'entre eux, il s'agit du second épisode de placement et pour $16,1 \%$, du troisième ${ }^{7}$. En moyenne, ils ont vécu 1,7 épisode de placement (écart-type $=0,8$ ) au cours de leur vie. La durée moyenne de l'épisode actuelle de placement $^{8}$ est de 32,3 mois (écart-type de 27,2). Quarante et un pour cent des enfants sont placés depuis moins de deux ans, $35,7 \%$ depuis une période de deux à trois ans et $23,2 \%$ depuis quatre ans et plus.

Les principales raisons de placement invoquées par les parents sont des problèmes de santé mentale ou physique du parent $(38,2 \%)$, d'abus physique, sexuel ou de négligence $(25,4 \%)$, des problèmes de consommation (drogue ou alcool) du parent $(18,2 \%)$, des problèmes de comportements de l'enfant $(16,4 \%)$ et, finalement, dans un cas le refus du parent d'assumer les soins ou la garde de l'enfant. La majorité des parents $(67,2 \%)$ disent que le retour de l'enfant se fera dans plus d'un an et $32,8 \%$ des parents rapportent que l'enfant sera de retour dans 12 mois et moins.

\section{RÉSULTATS}

\section{La situation quant au maintien des liens}

Les résultats présentés dans la figure 1 indiquent que $19 \%$ des parents reçoivent la visite de leur enfant plus de quatre fois par

6 Pour les parents qui ont plusieurs enfants placés, l'étude ne porte que sur un seul des enfants. Dans deux situations de placement, nous avons rencontré le père et la mère, ce qui explique un nombre de parents supérieur au nombre d'enfants placés.

7 Le nombre de placements vécus par les enfants ne renvoie pas à des changements de ressource d'accueil, mais bien à des placements effectués à partir du milieu familial.

8 Un épisode de placement représente la période de temps écoulée depuis le départ du milieu familial. Pour certains enfants, cet épisode de placement s'est déroulé dans le même milieu d'accueil; pour d'autres, il y a eu plusieurs ressources. Nous calculons la durée totale du séjour en milieu d'accueil, sans retour dans le milieu familial. 
mois, $25,9 \%$ de trois à quatre fois par mois, 37,9 \% deux fois par mois et $17,2 \%$ une fois par mois et moins. En ce qui a trait au nombre de jours (période de 24 heures) que l'enfant a passés chez son parent au cours du mois qui précède la collecte des données, on observe qu'en moyenne les parents ont accueilli leur enfant durant 3,7 jours (écart-type $=4,0$ ).

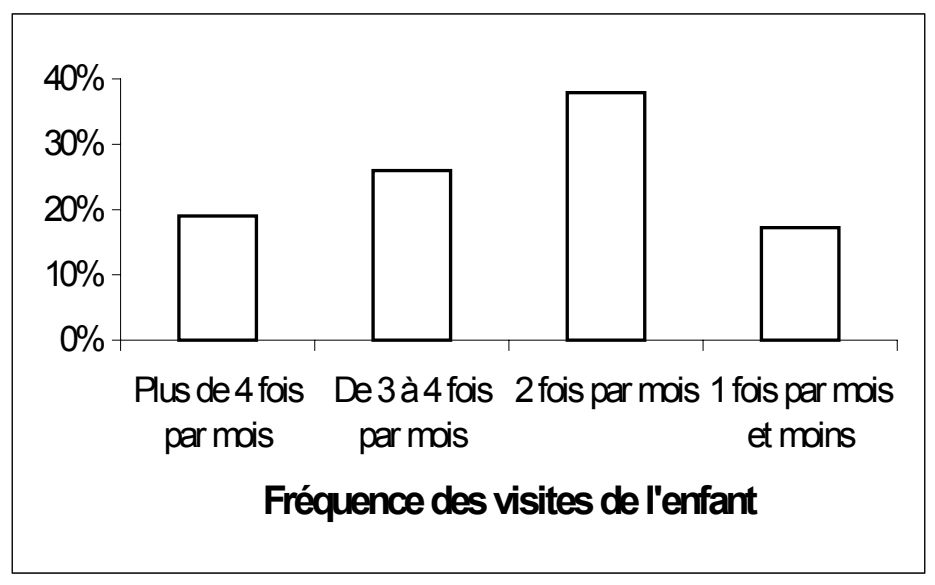

\section{Figure 1 \\ Proportion de parents en fonction de la fréquence des visites de l'enfant}

La perception du parent concernant le déroulement des visites a été évaluée : la majorité $(87,9 \%)$ considèrent qu'elles se passent bien; toutefois, près de $40 \%$ rapportent des difficultés liées à leur organisation. Ces difficultés sont liées au transport (difficultés à trouver un moyen de transport, frais associés au transport), aux relations difficiles avec les parents d'accueil ou avec l'intervenant social (négociation du plan et des horaires de visites), au fait que l'enfant refuse de venir à la maison et aux relations difficiles avec leur ex-conjoint.

\section{Variables associées à la fréquence et à la durée des visites}

Ainsi que le montre le tableau 2, aucune caractéristique des parents n'est associée de façon significative à la fréquence ou à la durée des visites de l'enfant dans son milieu familial. Toutefois, on observe, sur le plan des caractéristiques des familles, que les parents en contact avec moins de milieux d'accueil reçoivent plus souvent la visite de leur enfant ( $r h o=-0,266, p=0,044, n=54$ ). 
Tableau 2

Variables associées à la fréquence et à la durée des visites de l'enfant dans sa famille

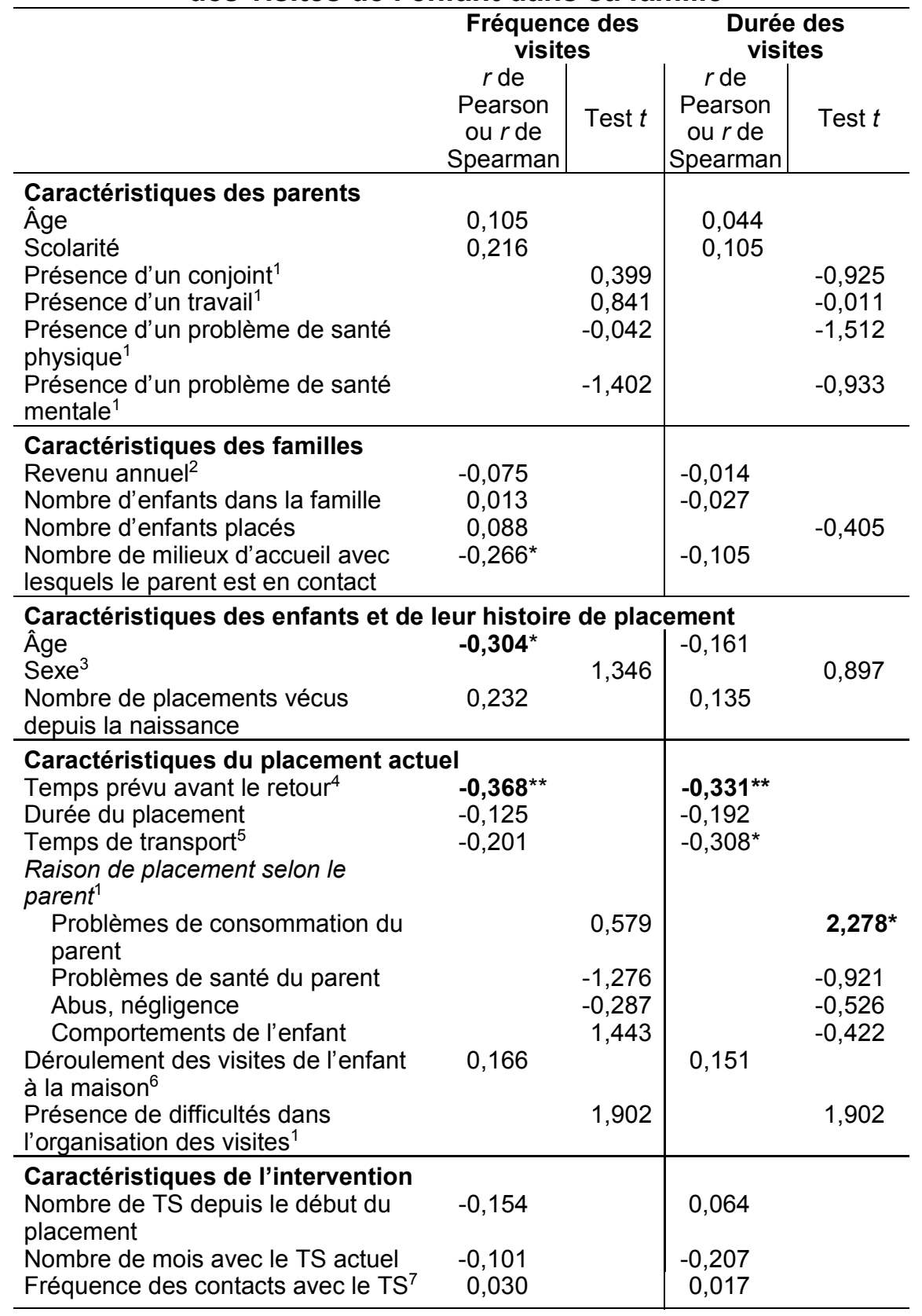


${ }^{*} \mathrm{p}<0,05,{ }^{* *} \mathrm{p}>0,01$

1. Non $=0$, Oui $=1$.

2. $1000 \$$ à $5999 \$=1,6000 \$$ à $11999 \$=2,12000 \$$ à $19999 \$=3$, $20000 \$$ à $24999 \$=4$ et $25000 \$$ et plus $=5$.

3. Masculin $=0$, Féminin $=1$.

4. Trois mois et moins $=1$, Dans 4 à 6 mois $=2$, Dans 7 à 12 mois $=3$, Dans plus de 12 mois $=4$.

5. Moins de $15 \mathrm{~min}=1$, Entre 15 et $30 \mathrm{~min}=2$, Entre 31 et $60 \mathrm{~min}=3$, Plus de $60 \min =4$.

6. Très mal $=1$, Mal $=2$, Bien $=3$, Très bien $=4$.

7. Moins d'une fois aux 3 mois $=1$, Moins d'une fois aux 3 mois, mais moins d'une fois par mois $=2$, Une fois par mois $=3$, Plus d'une fois par mois $=4$.

Parmi les caractéristiques personnelles des enfants et celles concernant l'histoire de placement, seul l'âge est associé aux visites. En effet, les enfants plus jeunes visitent leurs parents plus fréquemment que les enfants plus âgés $(r=-0,304, p=0,023, n=$ 56). Toutefois, aucune association n'apparaît entre l'âge des enfants et la durée des visites dans le milieu familial. Quant aux caractéristiques de la situation actuelle de placement, on constate que plus le retour de l'enfant est imminent (selon les parents), plus les visites sont fréquentes ( $r h o=-0,368, p=0,004, n=58$ ) et plus les séjours sont longs (rho $=-0,331, p=0,011, n=58$ ).

On observe également que les parents qui rapportent un temps de transport moins long reçoivent leur enfant plus de jours par mois $(r=-0,308, p=0,022, n=55)$. Par ailleurs, il n'y a aucune association entre les différentes raisons de placement et la fréquence des visites de l'enfant dans son milieu familial. Toutefois, en comparant les enfants placés en raison des problèmes de consommation de leur parent et les enfants placés pour d'autres raisons, on observe qu'ils passent moins de jours par mois dans leur famille $(2,2$ jours comparativement à 4,2 jours $[t=2,278, p=$ $0,027, \mathrm{dl}=54])$.

Bien qu'elle ne soit pas statistiquement significative $(t=1,902, p=$ $0,06, \mathrm{dl}=54$ ), on constate une différence dans la durée des visites de l'enfant dans sa famille entre le groupe de parents qui rapportent des problèmes dans l'organisation des visites et ceux qui n'en mentionnent pas. Dans le mois qui a précédé la collecte des données, les parents ayant rapporté des problèmes ont reçu la visite de leur enfant durant 3 jours comparativement à 4,5 jours pour les autres parents. Rappelons que les principales difficultés 
mentionnées par les parents sont les problèmes de transport, les relations avec les parents d'accueil et les intervenants, le refus de l'enfant de venir chez ses parents et les relations difficiles avec l'ex-conjoint.

\section{Résultats de l'analyse multivariée}

Afin d'avoir une meilleure compréhension des variables associées aux visites de l'enfant dans son milieu familial une analyse de régression multiple a été effectuée pour chacune des variables dépendantes (durée et fréquence des visites). II est à noter que certaines variables ordinales qui ne présentaient pas une distribution normale ont été transformées en variables dichotomiques ${ }^{9}$. De plus, afin de conserver la variable " raisons de placement ", il a fallu la transformer en créant une nouvelle variable dichotomique (absence/présence) avec chacune des catégories de la variable. Dans les analyses, trois des quatre variables ainsi créées doivent être entrées. La variable qui n'est pas entrée constitue la catégorie de référence à partir de laquelle les autres seront comparées ${ }^{10}$. La catégorie de référence choisie est le placement pour des raisons de problèmes de consommation du parent. Ce qui signifie que les résultats obtenus sur les autres variables en lien avec la raison de placement (négligence ou abus, comportements des enfants, problèmes de santé des parents) doivent être interprétés en comparaison avec cette catégorie.

Étant donné la taille de l'échantillon, toutes les variables dépendantes retenues dans cette étude n'ont pu être considérées simultanément dans une seule analyse de régression. Afin de sélectionner les variables indépendantes à inclure, toutes les variables de chacune des catégories (caractéristiques des parents, des familles, de l'enfant et de son histoire de placement,

9 Les variables transformées en variables dichotomiques aux fins d'analyse sont : le nombre de milieux d'accueil avec lesquels le parent est en contact; la fréquence des contacts avec les parents d'accueil; le nombre d'intervenants depuis le début du placement; le nombre de mois avec l'intervenant actuel et la fréquence des contacts avec l'intervenant.

${ }^{10}$ Schroeder, Sjoquist et Stephan (1989) précisent que le choix de la catégorie de référence n'a pas d'importance et qu'il n'influence pas les résultats. 
du placement actuel et de l'intervention) ont été soumises à une analyse de régression multiple pour déterminer les variables les plus fortement associées à la durée ou à la fréquence des visites, et ce, en tenant compte de plusieurs variables à la fois. Par la suite, les variables dont la contribution est apparue plus importante $^{11}$ dans chacune des catégories ont été insérées dans un modèle de régression final. La méthode d'analyse de régression standard qui consiste à entrer en même temps dans l'équation l'ensemble des variables a été utilisée.

\section{Régression multiple : la fréquence des visites}

Les analyses de régression effectuées avec les différentes catégories de variables indépendantes mettent en exergue cinq variables plus fortement associées à la fréquence des visites: le nombre d'enfants placés dans la famille, le nombre de milieux d'accueil avec lesquels le parent est en contact, l'âge de l'enfant, le temps prévu avant le retour de l'enfant ainsi que l'évaluation du déroulement des visites faite par le parent. Le tableau 3 présente les résultats de l'analyse de régression effectuée à partir de ces variables. L'ensemble de ces variables permet d'expliquer $21 \%$ de la variance de la fréquence des visites de l'enfant dans son milieu familial $\left(R^{2} a d j .=0,21\right)$. Les coefficients bêta $(\beta)$ standardisés indiquent que les variables qui prédisent le mieux la fréquence des visites de l'enfant dans sa famille sont le nombre de milieux d'accueil avec lesquels le parent est en contact $(\beta=-0,297)$, le temps prévu avant le retour de l'enfant $(\beta=-0,271)$ et l'âge de l'enfant $(\beta=-0,231)$. Les deux autres variables semblent avoir une contribution plus faible à la prédiction de la variable dépendante (nombre d'enfants placés, $\beta=0,154$ et déroulement des visites, $\beta=0,111)$. Toutefois, les valeurs des test $t$ pour les coefficients de régression de ces différentes variables indiquent que la contribution du nombre de milieux d'accueil avec lesquels le parent est en contact, le temps prévu avant le retour de l'enfant dans sa famille et l'âge de l'enfant sont significatifs. En raison de la taille de l'échantillon et de sa constitution non aléatoire, un seul de signification inférieur à 0,10

${ }^{11}$ Un seuil de sélection de 0,1 a été retenu pour ces analyses. Ce seuil de signification statistique est utilisé comme un indicateur permettant de comparer les variables et de sélectionner celles qui semblent avoir une contribution plus importante. 
$(p<0,10)$ a été retenu pour déterminer les variables qui ont une contribution significative à la prédiction de la variable dépendante.

Tableau 3

Caractéristiques du modèle de régression multiple de l'analyse de la fréquence des visites de l'enfant dans sa famille

\begin{tabular}{|c|c|c|c|c|c|}
\hline $\begin{array}{l}R^{2} \\
R^{2} \text { ajusté } \\
F(5,50)=\end{array}$ & $\begin{array}{l}0,282 \\
0,210 \\
92 p<0,01\end{array}$ & & & & \\
\hline & & $\begin{array}{l}\text { Non } \\
\text { standardisé }\end{array}$ & $\begin{array}{l}\text { ariables de l' } \\
\text { Standardisé }\end{array}$ & ation & \\
\hline Variables & & B & Bêta $\beta$ & $t$ & Sig. $t$ \\
\hline \multicolumn{2}{|c|}{$\begin{array}{l}\text { Nombre de milieux d'accueil } \\
\text { avec lesquels le parent est } \\
\text { en contact }\end{array}$} & $-0,867$ & $-0,297$ & $-2,116$ & 0,039 \\
\hline \multicolumn{2}{|c|}{$\begin{array}{l}\text { Temps prévu avant le retour de } \\
\text { l'enfant }\end{array}$} & $-0,381$ & $-0,271$ & 0,894 & 0,034 \\
\hline \multicolumn{2}{|c|}{ Âge de l'enfant } & $-0,100$ & $-0,231$ & $-1,869$ & 0,067 \\
\hline \multicolumn{2}{|c|}{ Nombre d'enfants placés } & 0,235 & 0,154 & 1,081 & 0,285 \\
\hline \multicolumn{2}{|c|}{$\begin{array}{l}\text { Déroulement des visites de } \\
\text { l'enfant à la maison }\end{array}$} & 0,194 & 0,111 & 0,894 & 0,376 \\
\hline \multicolumn{2}{|c|}{ Constante } & 4,473 & & 4,812 & 0,000 \\
\hline
\end{tabular}

\section{Régression multiple : la durée des visites}

Les analyses de régression effectuées avec les différentes catégories de variables indépendantes permettent de relever huit variables : la présence d'enfants à la maison, le temps de transport entre les deux milieux de vie de l'enfant, le temps prévu avant le retour de l'enfant, l'évaluation que fait le parent du déroulement des visites et les difficultés éprouvées ainsi que l'identification de la principale raison de placement (problèmes de santé physique ou mentale du parent, situations d'abus ou de négligence et comportements de l'enfant). Ces variables permettent d'expliquer $31 \%$ de la variance de la durée des visites de l'enfant dans son milieu familial $\left(R^{2}\right.$ adj. $\left.=0,31\right)$ (voir tableau 4$)$. L'étude des coefficients standardisés (bêta $\beta$ ) montre que les variables qui contribuent le plus à la prédiction de l'augmentation de la durée des 
visites de l'enfant dans sa famille sont le fait que l'enfant soit placé en raison de problèmes d'abus ou de négligence $(\beta=0,445)$, la présence de difficultés dans l'organisation des visites $(\beta=-0,370)$ et le placement de l'enfant pour des problèmes de santé physique ou mentale du parent $(\beta=0,369)$ ainsi que l'évaluation du déroulement des visites $(\beta=0,284)$. Les autres variables semblent avoir une contribution plus faible (le placement pour des problèmes de comportement de l'enfant, $\beta=0,249$; la présence d'enfants à la maison, $\beta=0,236$; le temps prévu avant le retour de l'enfant, $\beta=-0,234$ et le temps de transport, $\beta=-0,234$ ).

Les valeurs des test $t$ pour les coefficients de régression de ces différentes variables indiquent que la contribution de toutes les variables est significative $(p<0,10)$, mis à part celle de la variable placement en raison des problèmes de comportement de l'enfant.

Tableau 4

Caractéristiques du modèle de régression multiple de l'analyse de la durée des visites de l'enfant dans sa famille

\begin{tabular}{|c|c|c|c|c|}
\hline $\begin{array}{ll}R^{2} & 0,422 \\
R^{2} \text { ajusté } & 0,312 \\
F(8,42)=3,83 & p<0,01\end{array}$ & & & & \\
\hline & $\begin{array}{l}\text { Variables } \\
\text { Non } \\
\text { standardisé }\end{array}$ & $\begin{array}{l}\text { de l'équation } \\
\text { Standardisé }\end{array}$ & & \\
\hline Variables & B & Bêta $\beta$ & $t$ & Sig. $t$ \\
\hline $\begin{array}{l}\text { Présence de difficultés dans } \\
\text { l'organisation des visites }\end{array}$ & $-1,887$ & $-0,370$ & $-2,805$ & 0,008 \\
\hline $\begin{array}{l}\text { Raison du placement : } \\
\text { - problèmes de santé }\end{array}$ & 1880 & 0.369 & 2.143 & 0.038 \\
\hline $\begin{array}{l}\text { physique ou mentale } \\
\text { - abus ou négligence }\end{array}$ & 2,481 & 0,445 & $\begin{array}{l}2,140 \\
2,571\end{array}$ & 0,014 \\
\hline $\begin{array}{l}\text { - problèmes de comportement } \\
\text { de l'enfant }\end{array}$ & 1,625 & 0,249 & 1,403 & 0,168 \\
\hline $\begin{array}{l}\text { Déroulement des visites de } \\
\text { l'enfant à la maison }\end{array}$ & 1,096 & 0,284 & 2,170 & 0,036 \\
\hline $\begin{array}{l}\text { Présence d'enfants à la maison } \\
\text { Temps de transport }\end{array}$ & $\begin{array}{r}1,194 \\
-0,613\end{array}$ & $\begin{array}{r}0,236 \\
-0,234\end{array}$ & $\begin{array}{r}1,678 \\
-1,889\end{array}$ & $\begin{array}{l}0,101 \\
0,066\end{array}$ \\
\hline $\begin{array}{l}\text { Temps prévu avant le retour de } \\
\text { l'enfant }\end{array}$ & $-0,688$ & $-0,234$ & $-1,919$ & 0,062 \\
\hline Constante & 3,048 & & 1,674 & 0,102 \\
\hline
\end{tabular}




\section{DIscussion}

La fréquence des visites entre les parents et les enfant placés observée dans cette étude est plus élevée que ce que l'on retrouve habituellement dans la littérature, seulement $17,2 \%$ des parents recevant la visite de leur enfant une fois par mois et moins. Cette proportion est de $24 \%$ dans l'étude ontarienne de Kufeldt et collab. (1995) et de $57 \%$ dans l'étude québécoise menée par Simard et collab. (1998). Cette différence pourrait être attribuable à l'un des critères de sélection de notre échantillon, soit que le parent ait eu au moins un contact au cours des six derniers mois. La présente étude n'a pas permis de décrire les situations de placement dans lesquelles on observe une rupture de contact ou des contacts plus irréguliers entre les parents et leur enfant placé. Malgré cette limite, nous croyons que cette étude a favorisé une meilleure compréhension de la complexité des facteurs influençant le maintien des liens entre les parents et l'enfant placé. Nous devons toutefois mentionner que la présente étude a été menée dans une perspective corrélationnelle et qu'aucun lien de causalité ne peut être dégagé des observations effectuées.

En ce qui concerne la fréquence des visites de l'enfant, on constate que celles-ci sont plus fréquentes lorsque le parent est en contact avec un seul milieu d'accueil, lorsque l'enfant placé est plus jeune et que l'on prévoit rapidement son retour dans sa famille. Ces trois variables expliquent $21 \%$ de la variance observée sur le plan de la fréquence des visites de l'enfant chez ses parents. Les parents qui sont en contact avec un nombre moins grand de milieux d'accueil (les parents ayant un seul enfant placé ou les parents ayant plus d'un enfant placé mais dans la même ressource, $N=38$ ) reçoivent la visite de leur enfant plus fréquemment que les parents qui ont plus d'un enfant placé dans des ressources différentes $(N=20)$. Ce résultat soulève des questions sur la séparation des fratries lors d'un placement et son impact sur le maintien des liens familiaux entre les frères et sœurs, mais également avec les parents. On observe également une fréquence moins élevée de contacts pour les enfants plus vieux. Ce résultat est semblable à ce qui est observé par O'Higgins (1996). Nous pouvons émettre l'hypothèse que certains 
enfants plus âgés expriment leur point de vue concernant les visites chez leurs parents. En effet, certains parents ont mentionné le refus de l'enfant comme un aspect problématique lors de l'organisation des visites. Cela soulève l'importance de la perspective des enfants en ce qui concerne le maintien des liens et la nécessité de s'intéresser à leur point de vue dans les futurs travaux de recherche sur cette question. Tout comme dans l'étude de Fanshel (1982), la prévision du retour de l'enfant dans son milieu familial est associée positivement au maintien des liens entre l'enfant placé et ses parents. Le maintien des liens parentenfant ne répond pas aux mêmes objectifs dans les cas de placements à long terme et dans ceux où l'on prévoit le retour de l'enfant. Cela illustre l'importance de la planification du retour de l'enfant, mais encore plus celle de la mise en place d'un échéancier plus précis. Dans notre étude, la majorité des parents ont mentionné le retour des enfants avant leur majorité. Toutefois, peu de parents ont été capables d'indiquer clairement le moment du retour. Cette absence de prévision claire peut amener une démobilisation de la part des parents, mais également des intervenants et des parents d'accueil qui ne travailleront plus directement dans l'optique d'une réinsertion familiale.

Sur le plan de la durée des visites, c'est-à-dire le nombre de jours que l'enfant a passés chez ses parents dans le mois qui précède la collecte des données, on constate dans les analyses bivariées que les enfants qui passent plus de temps chez leurs parents ne sont pas placés en raison des problèmes de consommation du parent, qu'ils sont placés plus près de leur milieu familial et que le temps prévu avant leur retour est plus court. Dans les analyses de régression multiple, l'importance de certaines variables liées spécifiquement aux visites est ressortie, soit la présence de problèmes dans l'organisation des visites et l'évaluation de difficultés lors de leur déroulement. Comme nous l'avons vu précédemment, la fréquence des visites est dictée par les disponibilités du parent (nombre d'enfants placés et séparation ou non de la fratrie), l'âge de l'enfant et la prévision du retour. Ces variables influencent la mise en place du plan de visites (par exemple, toutes les fins de semaine ou une fin de semaine sur deux). Toutefois, les variables associées à la durée des visites indiquent que le climat dans lequel elles s'organisent et se déroulent agit directement sur le temps passé par l'enfant dans sa famille. Ce résultat suggère une 
certaine marge de manœuvre dans l'actualisation de cette planification (les heures d'arrivée et de départ, les visites lors des journées de congé scolaire, l'annulation de certaines visites) qui est associée à d'autres aspects du placement, comme les difficultés de transport, les relations avec les intervenants et les parents d'accueil, de même que les problèmes personnels des parents.

Les rapports de recherche consultés indiquent également une association entre la présence de difficultés liées au transport et la faible fréquence de contacts parents-enfants (Fanshel, 1982; Jenkins et Norman, 1975; Kufeldt et collab., 1996). Dans notre étude, parmi les vingt-deux parents qui rapportent des difficultés dans l'organisation des visites, neuf mentionnent des difficultés liées au transport (trouver un moyen de transport ou en supporter les frais). Compte tenu du petit nombre de parents dans notre échantillon, il est impossible de vérifier le poids de cette variable d'un point de vue quantitatif. Le temps de transport comme variable affectant négativement le maintien des liens entre l'enfant placé et ses parents attire l'attention sur l'importance d'actualiser, à tous les niveaux, les nouvelles façons d'envisager les placements. En effet, si l'on désire favoriser la présence des parents lors du placement des enfants, on doit éviter de placer les enfants dans des milieux d'accueil éloignés de leur milieu familial.

Certains parents mentionnent vivre au quotidien des difficultés dans leurs discussions avec les parents d'accueil et les intervenants en ce qui a trait à l'organisation des visites. On peut penser que, même si l'importance du maintien des liens est reconnue par tous, sa concrétisation dans la réalité peut présenter certaines difficultés. Les parents mentionnent des difficultés liées aux négociations des aspects concrets des visites : le transport, les heures de départ et d'arrivée, les annulations, les visites lors d'activités spéciales (fêtes, congés scolaires).

Finalement, il est difficile de préciser si c'est le fait que les visites se déroulent moins bien qui a un effet sur la fréquence ou si c'est le fait qu'elles soient moins fréquentes qui les rend plus difficiles. Toutefois, de nombreux parents ont mentionné, au moment de l'entrevue, que les relations avec l'enfant et la discipline entraînaient certaines difficultés lors des visites. Les parents dont les 
enfants sont placés éprouvent souvent des difficultés sur le plan des habiletés parentales. II apparaît donc important d'aider les parents et de les outiller afin que les contacts avec leur enfant soient plus adéquats et agréables.

La poursuite des travaux dans ce domaine devrait tenir compte de l'importance du climat dans lequel s'inscrit le maintien des liens, donc viser à comprendre les facteurs qui influencent la qualité des contacts entre les parents et l'enfant ainsi que ceux qui facilitent les relations entre les adultes qui prennent soin de l'enfant placé (parents, parents d'accueil et intervenants).

Marie-Andrée POIRIER Institut de recherche pour le développement social des jeunes Centre jeunesse de Montréal

\author{
Marie SIMARD \\ Directrice \\ École de service social \\ Université Laval \\ Jacques VACHON \\ Professeur \\ École de service social \\ Université Laval, Québec
}

\title{
Références bibliographiques
}

BILSON, A. et R. BARKER (1995). «Parental Contact with Children Fostered and in Residential Care After the Children Act 1989 », British Journal of Social Work, vol. 25, p. 367-381.

BLOck, N.M. et A.S. LIBOWITZ (1983). Recidivism in Foster Care, New York, McGraw-Hill.

Bullock, R., K. Hosie, M. LitTle et S. Millham (1990). «The Problems of Managing the Family Contacts of Children in Residential Care », British Journal of Social Work, vol. 20, p. 591-610. 
CANTOS, L.C., L.T. GRIES et V. SlIS (1997). «Behavioral Correlates of Parental Visiting During Family Foster Care », Child Welfare, vol. 76, n०2, p. 309-329.

ClifFE, D. et D. BerRIDGe (1991). Closing Children's Homes : An End to Residential Childcare ? Londres, National Children Bureau.

Davies, I.P., J. LandsVerk, R. NeWton et W. Ganger (1996). «Parental Visiting and Foster care Reunification », Children and Youth Services Review, vol. $18, \mathrm{n}^{\text {os }} 4-5$, p. 363-382.

Fanshel, D. (1982). On the Road to Permanency. An Expanded Date Base for Service to Children in Foster Care, New York, Columbia University School of Social Work and Child Welfare League of America.

FANSHEL, D. et E. SHINN (1978). Children in Foster Care : a Longitudinal Investigation, New York, Columbia University Press.

Fein, E., A. Maluccio, J.V. Hamilton et D.E. Ward (1983). After Foster Care: Outcomes of Permanency Planning for Children, New York, Child Welfare League of America.

Galaway, B., R. Nutter et J. Hudson (1994). « Birth Parent Participation in Treatment Foster Family Care », dans B. McKenzie (dir.), Current Perspectives on Foster Family Care, Toronto, Wall and Emerson, p. 74-82.

GILlespie, J., B. BYRNE et L. WORKMAN (1995). "An Intensive Reunification Program for Children in Foster Care », Child and Adolescent Social Work Journal, vol. 12, n 3, p. 213-218.

GoUVERNEMENT DU QUÉBEC (1998). Données opérationnelles au 31 mars 1998, Québec, ministère de la Santé et des Services sociaux.

Gruber, A. (1978). Children in Foster Care, New York, Human Science Press.

HESS, P. et H. PROCH (1993). "Visiting: the Hearth of Reunification », dans B.A. Pine, R. Warsh et A. Maluccio (dir.), Together Again: Family Reunification in Foster Care, Washington, Child Welfare League of America.

JenKINS, S. (1969). " Separation Experience of Parents Whose Children are in Foster Care », Child Welfare, vol. 48, p. 334-340.

Jenkins, S. et H. Norman (1975). Beyond Placement: Mothers View Foster Care, New York, Columbia University Press.

Jenkins, S. et H. Norman (1972). Filial Deprivation and Foster Care, New York, Columbia University Press. 
KRonA, D.A. (1980). Parents as A Treatment Partners in Residential Care, Child Welfare, vol. 59, $\mathrm{n}^{\circ}$ 2, p. 91-96.

KUfELDT, K., J.R. ARMSTRONG et M. DOROSH (1996). «Connection and Continuity in Foster Care », Adoption and Fostering, vol. 20, n 2, p. 1-7.

KufELdT, K., J.R. ARMSTRONG et M. DOROSH (1989). "In Care, in Contact ? », dans J. Hudson et B. Galaway (dir.), The State as a Parent, Kluwer Academic Publishers, p. 355-368.

LESSARD, C. (2000). Indicateurs repères sur l'application de la Loi sur la protection de la jeunesse, 1993-1994 à 1998-1999, Service des indicateurs et mesure de la performance, Direction de la gestion de l'information, ministère de la Santé et des Services sociaux.

Maluccio, A., L.W. AbRAmCZYCK et B. ThOMLinson (1996). "Family Reunification of Children in Out-of-Home Care: Research Perspectives ", Children and Youth Services Review, vol. 18, $\mathrm{n}^{\circ} 4-5$, p. 287-305.

MeCH, E.V. (1985). "Parental Visiting and Foster Placement », Child Welfare, vol. 64, no 1, p. 67-72.

Newcomb, P.R. (1986). Parental Visiting in Family Foster Care. Thèse de doctorat, Ann Arbor, Michigan University.

Neuman, W.L (1997). Social Research Methods. Qualitative and Quantitative Approaches, $3^{\mathrm{e}}$ éd., Boston, Allyn and Bacon.

O'HIGgINS, K. (1996). Disruption, Displacement, Discontinuity? Children in Care and Their Families in Ireland, Aldershot, Avebury.

OYSERMAN D. et R. BEBENSHITY (1992). "Keeping in Touch : Ecological Factors Related to Foster Care Visitation », Child and Adolescent Social Work Journal, vol. 9, n 6, p. 541-554.

PALMER, S. (1995). Maintaining Family Ties. Inclusive Practice in Foster Care, Washington, Child Welfare League of America.

PALMER, S. (1992). «Including Birth Families in Foster Care : a CanadianBritish Comparison », Children and Youth Services Review, vol. 14, p. 407-425.

PoIRIER, M.-A. (2000). L'implication parentale lors du placement. La perspective des parents, Thèse de doctorat non publiée, École de service social, Université Laval, Québec.

POIRIER, M.-A. (1998). « Le maintien des liens entre l'enfant placé et ses parents. Analyse critique de travaux de recherche », Revue canadienne de service social, vol. 15, n 1, p. 9-24. 
RAVIER, I. (1995). Le lien familial à l'épreuve du placement. Rapport de recherche, Communauté française de Belgique, ministère de l'Aide à la Jeunesse, Centre Droit et Sécurité d'Existence et Faculté de droit.

Richardson, G., B. Galaway, J. Hudson, R. Nutter et M. Hill (1995). « Birth Parent Participation in Treatment Foster Care Programs in North America and the United Kingdom », dans J. Hudson et B. Galaway (dir.), Child Welfare in Canada. Research and Policies Implications, p. 219-232.

SHROEDER, L.D., D.L. SJOQUIST et P.E. STEPHAN (1986). Understanding Regression Analysis. An Introductory Guide. Series: Quantitative Applications in the Social Sciences, vol. 57, Newbury Park et Londres, Sage Publications.

SIMARD, M., J. VACHON et S. BÉRUBÉ (1998). Les familles d'accueil pour les jeunes en difficulté au Québec. Étude comparative des familles spécifiques et non spécifiques, Québec, Centre de recherche sur les services communautaires, Université Laval.

SIMARD, M., J. VACHON et C. TARD (1991). La réinsertion familiale de l'enfant placé : Analyse de la perception des parents, Québec, Centre de recherche sur les services communautaires, Université Laval.

SteINHAUER, P.D. (1996). Le moindre mal. La question du placement de l'enfant, traduit de l'anglais par Denise Marchand, Montréal, Les Presses de l'Université de Montréal.

TABACHNICK, B.G. et L.S. FIDELL (1989). Using Multivariate Statistics, $2^{\mathrm{e}}$ éd., New York, Harper Collins Publishers.

VACHON, J. (1978). Les parents face au placement, Université de Sherbrooke et le Centre des services sociaux du Montréal métropolitain. 